\title{
Mechanisms of Survival and Resistance of Arthropods to Drought in Marine and Stream Habitats and Possible use of its Survival Mechanism for Human
}

\section{Hassan Vatandoost ${ }^{1,2 *}$}

${ }^{1}$ Department of Medical Entomology and Vector Control, School of Public Health, Tehran University of Medical Sciences, Tehran, Iran

${ }^{2}$ Department of Environmental Chemical Pollutants and Pesticides, Institute for Environmental Research, Tehran University of Medical Sciences, Tehran, Iran

*Corresponding author: Hassan Vatandoost, Department of Medical Entomology and Vector Control, School of Public Health, Tehran University of Medical Sciences, Tehran, Iran.
Received Date: January 15, 2021

Published Date: February 15, 2021

\section{Opinion}

Some invertebrates, when severely dehydrated, go into a state of suspended animation in which their metabolisms completely cease. When conditions are right, the insect come back to life. The sleeping fly called Polypedilum vanderplanki belong to the order of Diptera and Family of Chironomidae. They live mainly in Africa (Figure 1).

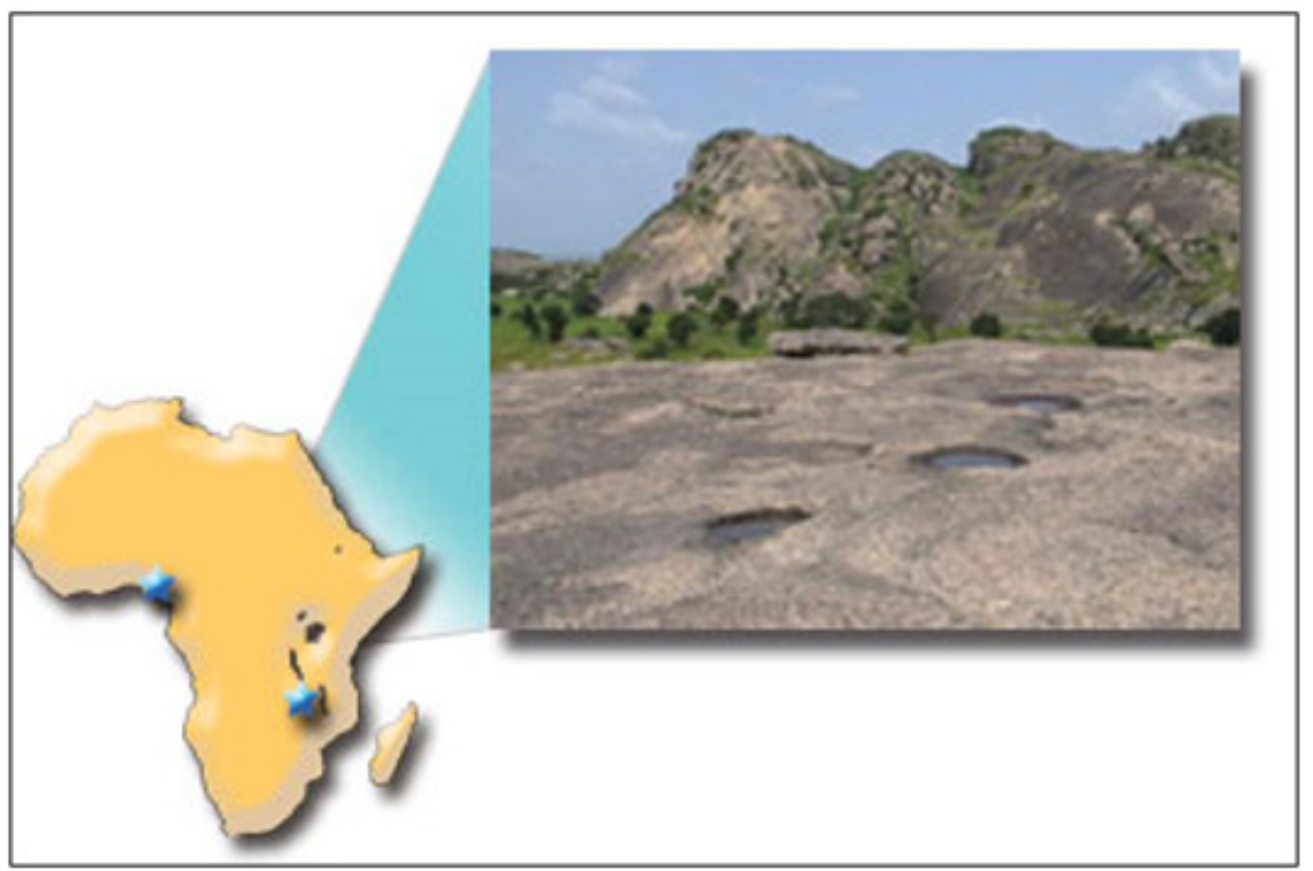

Figure 1: Breeding places of sleeping fly (Polypedilum vanderplanki). 
The life cycle of fly includes egg, larvae, pupae and adult (Figure 2). The larvae live in small tubular nests in the mud at the bottom of temporary pools that frequently dry out during the lifetime (Figure 3). The larvae of the African fly (Polypedilum vanderplanki) scrounge a living by eating detritus at the bottom of rain puddles but can survive up to 17 years of drought waiting for the next abundant rains. Figure 4 shows the male and female of this species with short life. Figure 5 shows the larvae of this species before and after sleeping. Since trehalose is the hemolymphatic sugar of insects, it represents 0.5 to $1 \%$ of the body lymph in larvae. However, during the process of desiccation, trehalose is rapidly synthesized to reach finally a content of $20 \%$ (Figure 6) (Figures 2-6).

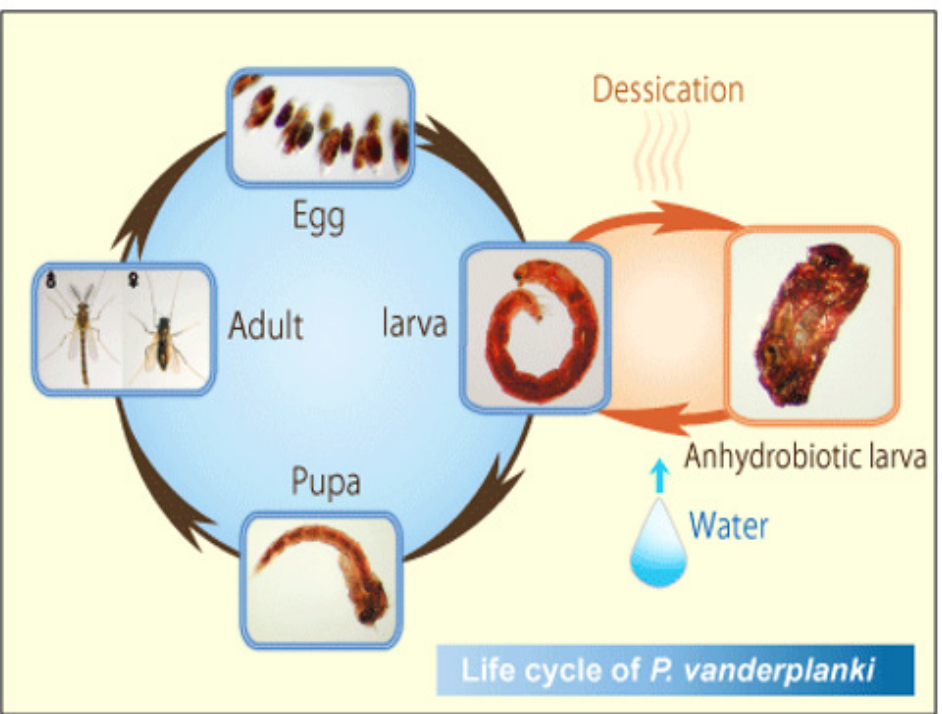

Figure 2: life cycle of sleeping fly (Polypedilum vanderplanki).

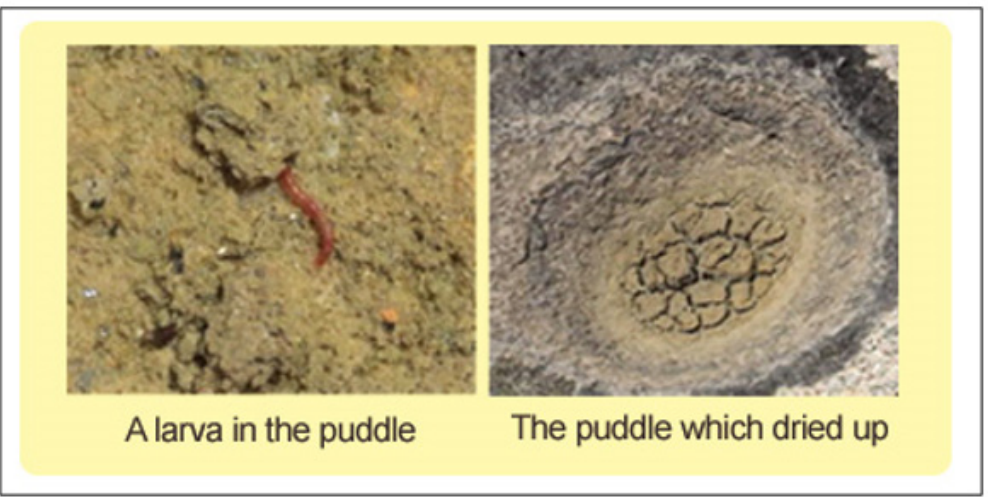

Figure 3: The larvae of sleeping fly (Polypedilum vanderplanki).

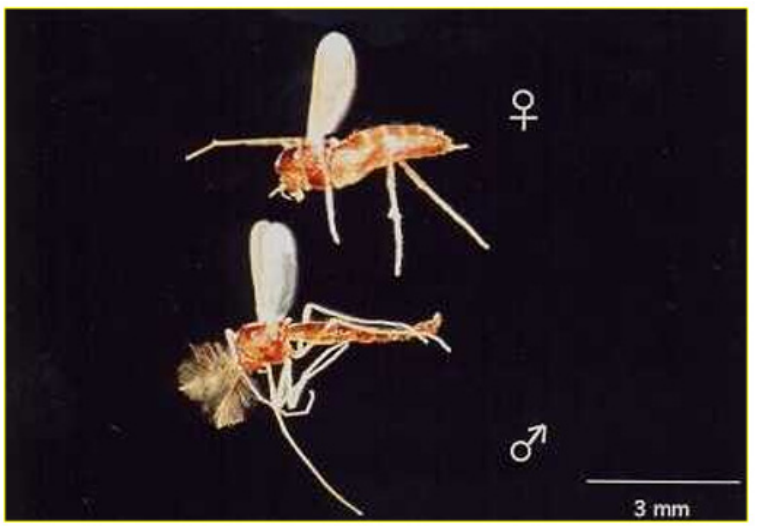

Figure 4: The male and female of sleeping fly (Polypedilum vanderplanki). 

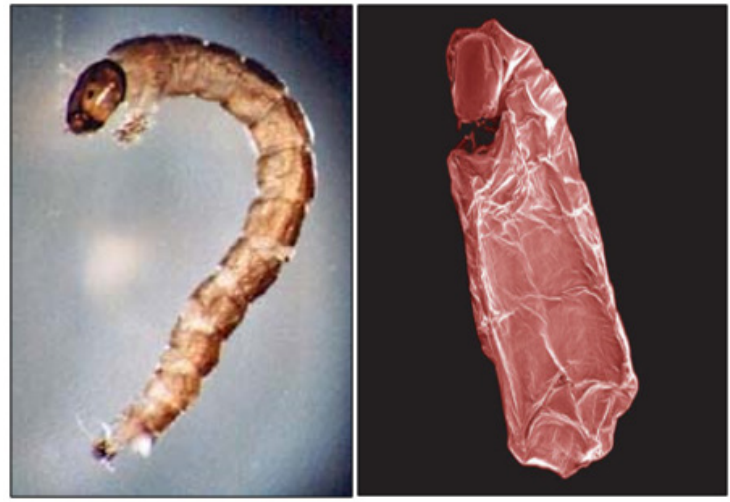

Figure 5: Larvae of sleeping fly (Polypedilum vanderplanki) before and after sleeping.

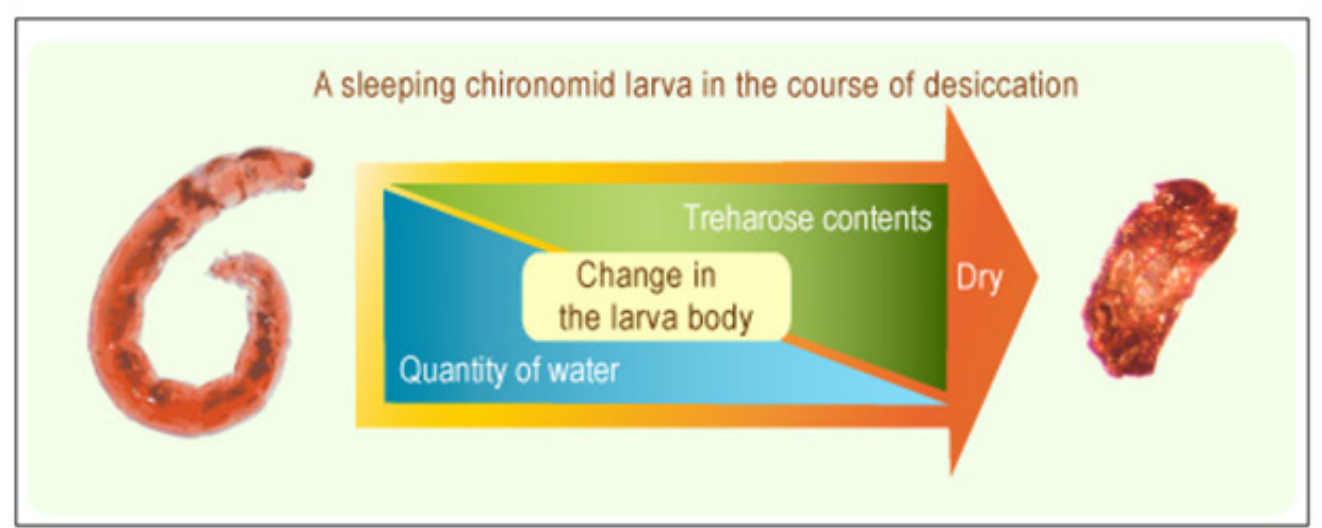

Figure 6: Process of desiccation sleeping fly (Polypedilum vanderplanki).

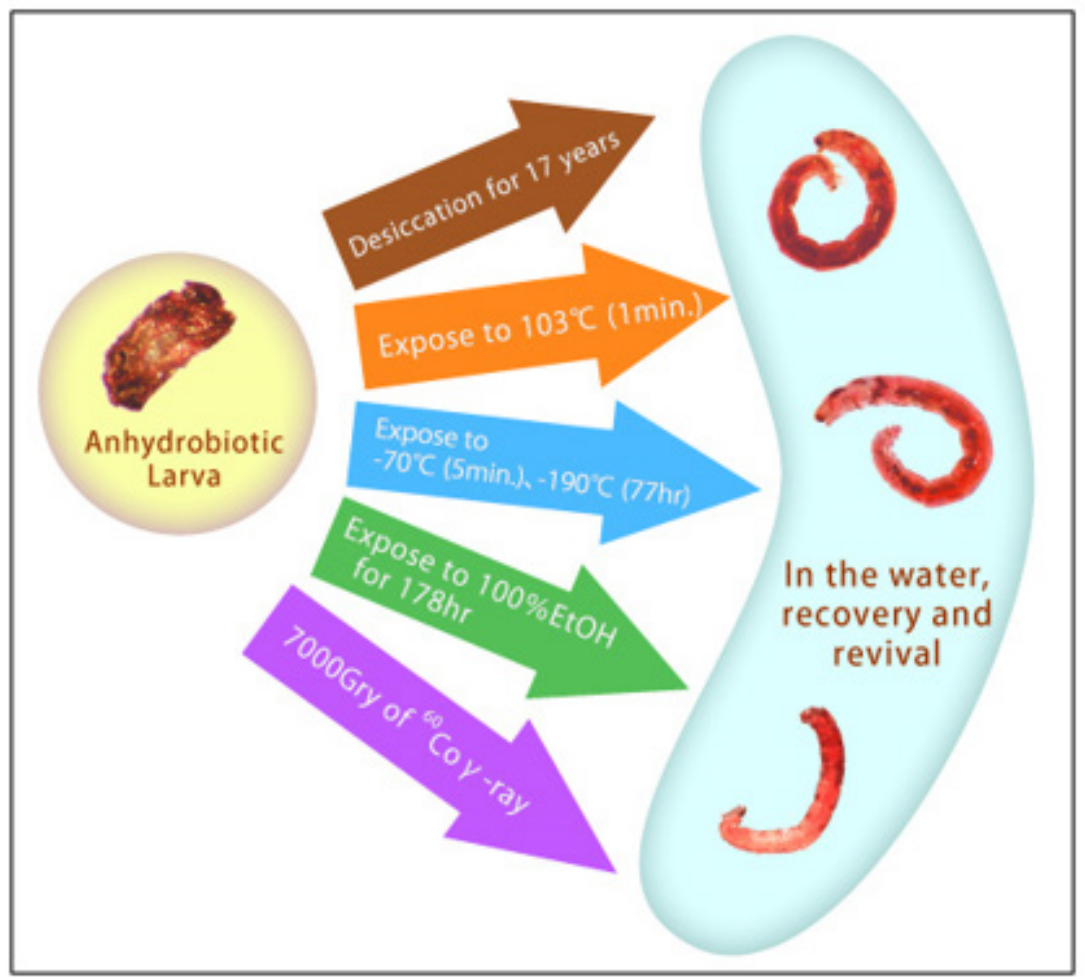

Figure 7: Survival of desiccation sleeping fly (Polypedilum vanderplanki) in different worse environmental conditions. 
Anhydobiotic larvae can survive for 17 years in desiccation of breeding places. If they expose to 103 degree centigrade for 1 minutes and then they were put in the normal water, they are able to recover. The experiments also showed that exposure to -190 degree centigrade for 77 hours and then expose to normal water, they will recover. In addition, exposure to ethanol 100\% for 178 hours doe nor effect on the mortality. Exposure to 7000 Gaba rate also does not effect on its life (Figure 7). The sleeping stage were transferee 400 kilometers from the earth and then put in the water, the recovered (Figure 8). If part of body including head is cut off and during andydobiosis, they transfer to the water, aging they alive, it seems that the nervous system of larvae are in different parts of body (Figure 9). There are Multi-center studies in the world for rearing of species to work on different aspects of its physiology for helping the human in adverse condition for survival (Figures 10,11). The mechanism of andydobiosis in the larvae is expression of specific genes to enhance the contents of trihalose in the body (Figure 12). Study on physiology and habits of this species will help the human to mimics its physiology in extreme and bad condition for survival (Figures7-12).

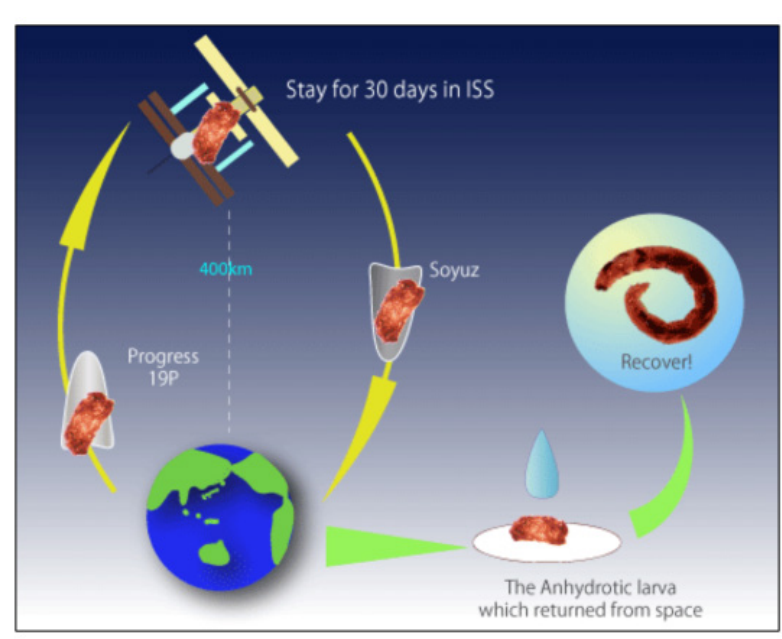

Figure 8: Transferring desiccation sleeping fly (Polypedilum vanderplanki) above the earth.

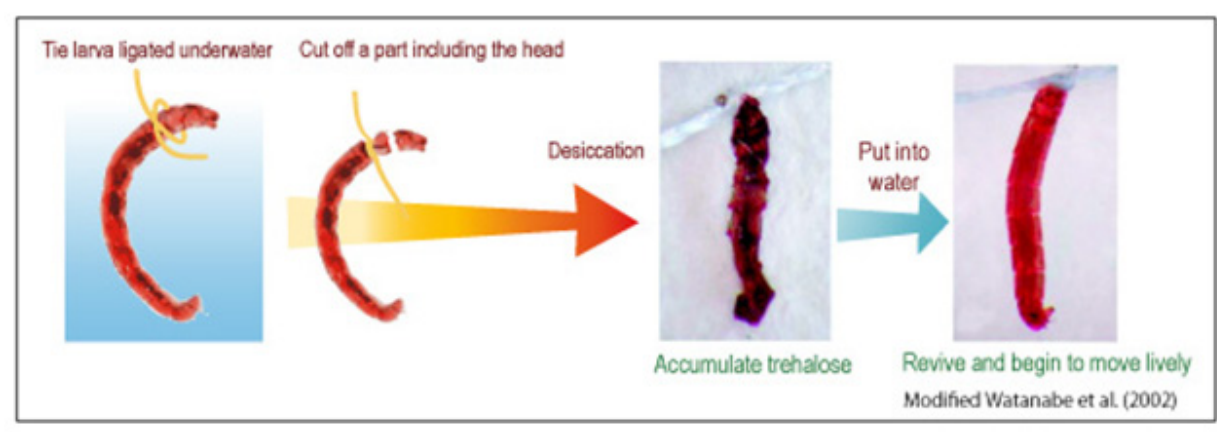

Figure 9: Cut off the head of sleeping fly (Polypedilum vanderplanki).

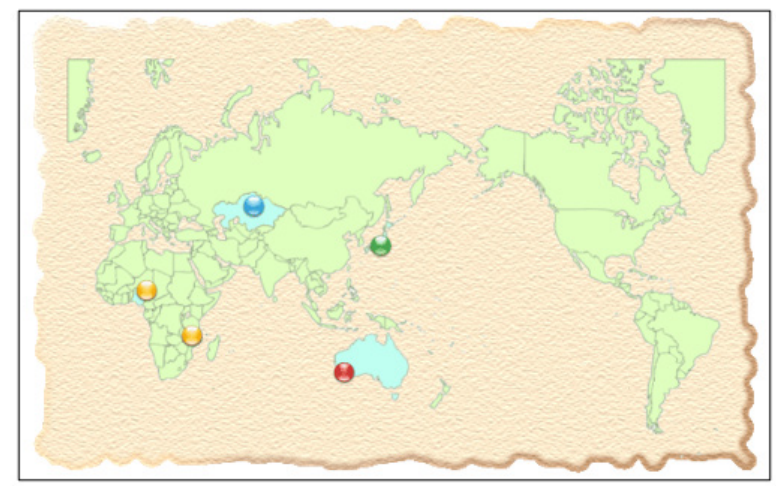

Figure 10: Multi-center study for rearing of sleeping fly (Polypedilum vanderplanki). 


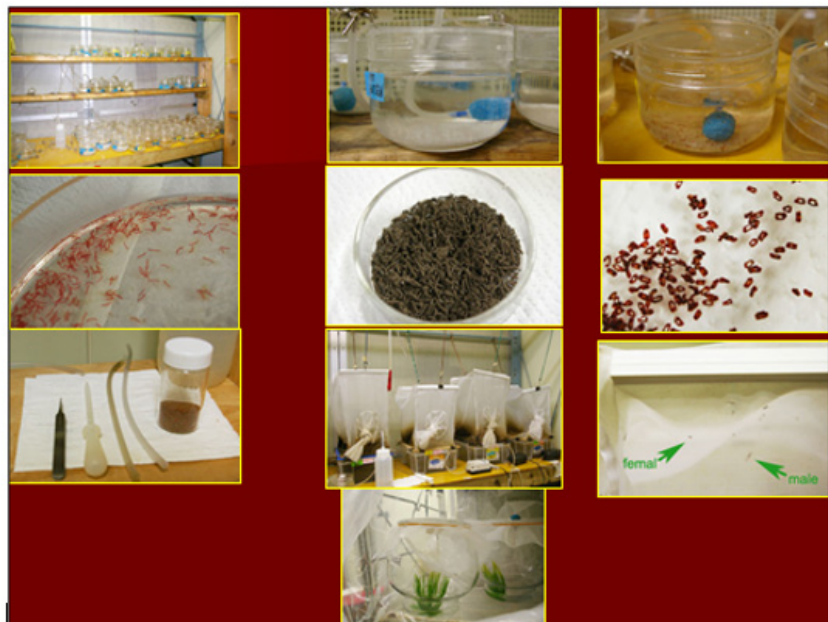

Figure 11: Breeding of sleeping fly (Polypedilum vanderplanki) in insectarium.

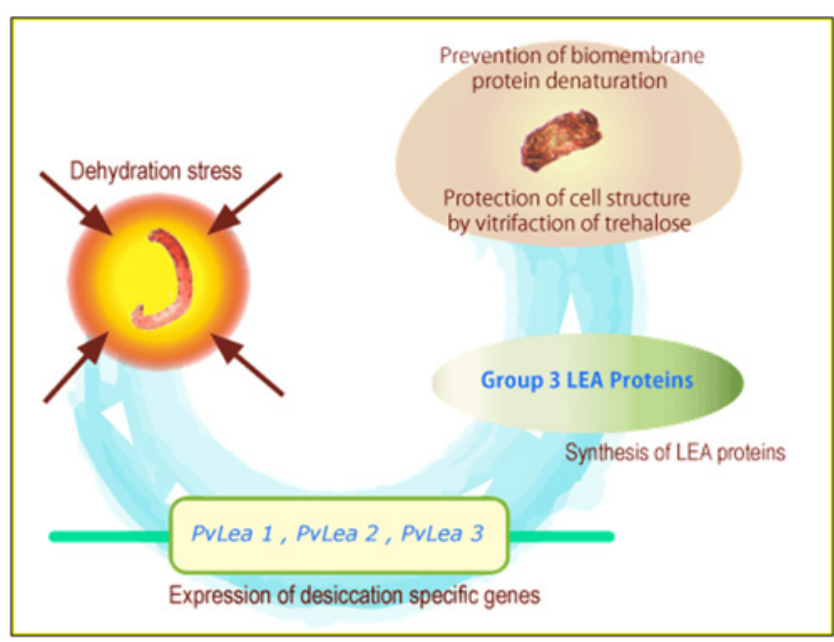

Figure 12: Expression of specific genes to enhance the contents of trehalose in the body of sleeping fly (Polypedilum vanderplanki).

\section{Acknowledgment}

None.

\section{Conflicts of Interest}

No conflict of interest. 